\title{
Photoluminescence of a Plasmonic Molecule
}

2 Da Huang, ${ }^{\dagger}$ Chad P. Byers, ${ }^{\dagger}$ Lin-Yung Wang, ${ }^{\dagger}$ Anneli Hoggard, ${ }^{\dagger}$ Ben Hoener, ${ }^{\dagger}$ Wei-Shun

3

4

5

6

7

8

9

10
Chang, ${ }^{\dagger}$ Christy F. Landes, ${ }^{\dagger, *}$ and Stephan Link, ${ }^{\dagger,+*}$

${ }^{\dagger}$ Department of Chemistry, Rice University

${ }^{\ddagger}$ Department of Electrical and Computer Engineering

Laboratory for Nanophotonics, Rice University, Houston, Texas 77005

*Corresponding authors, email: slink@rice.edu, cflandes@rice.edu 
1

2 Photoluminescent $\mathrm{Au}$ nanoparticles are appealing for bio-sensing and bio-imaging applications

\section{TOC FIGURE}

18

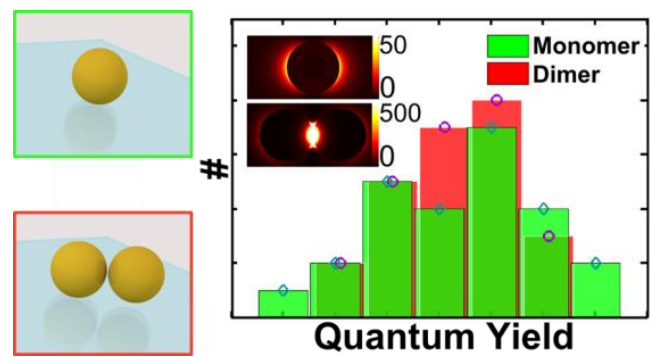


1

3 imaging down to the single molecule level. ${ }^{1,2}$ Compared to molecules, Au nanostructures have

4 much lower PL quantum yields (\# of photons emitted / \# of photons absorbed), but do not suffer

5 from photoblinking and irreversible photobleaching. In addition, due to their large absorption

6 cross sections and fast relaxation the PL brightness (\# of photons emitted / time) is comparable to

7 organic dyes ${ }^{3}$ while the PL does not easily photo-saturate with increasing excitation intensities. ${ }^{4}$

8 These properties make Au nanostructures attractive candidates in bio-sensing and bio-imaging

9 applications. $^{4,5}$ To maximize the potential of $\mathrm{Au}$ nanostructures as PL labels, a detailed

10 mechanistic understanding of the one-photon PL is first required.

To date, several mechanisms have been proposed based on experimental investigations of

12 the light emission from Au nanoparticles. These mechanisms include the radiative recombination

13 of electron-hole pairs via interband transitions ${ }^{6-10}$ and intraband transitions, ${ }^{11}$ the radiative decay

14 of surface plasmons, ${ }^{12-18}$ antenna-enhanced emission from surface ligands ${ }^{19,20}$ and intrinsic small

15 clusters (nanometer grain sizes), ${ }^{21}$ or emission through resonant electronic Raman scattering. ${ }^{22}$

16 Although the involvement of localized surface plasmons is generally accepted given the large PL

17 enhancement for nanostructures compared to bulk films, ${ }^{6,7,23,24}$ strong disagreement exists

18 whether the PL is simply enhanced by the local electric field or if the surface plasmons play a 19 more direct role.

20

To test the influence of the local electric field on the PL of Au nanostructures, we have

21 compared the PL from colloidal Au nanospheres (AuNS) and their dimers, representing the

22 simplest form of a plasmonic molecule. ${ }^{25,26}$ With separations much less than the constituent 
1

nanoparticle radii, strong capacitive coupling leads to greatly enhanced local electric fields. ${ }^{27-30}$ By using single-particle spectroscopy to eliminate sample inhomogeneities encountered in bulk measurements and employing a dimer enrichment procedure to ensure that enough AuNS dimers were measured for a quantitative, statistical analysis, we determined the PL quantum yield of individual AuNSs and strongly coupled AuNS dimers. We found that the PL quantum yield was not affected by the increased electric field of the dimers. These results, in combination with the spectral dependence of the PL from AuNS monomers and dimers, give further evidence to the mechanism of plasmon emission following hot electron generation.

\section{RESULTS AND DISCUSSION}

A colloidal sample with an enriched dimer population and without the presence of many larger aggregates that would negatively interfere in the single-particle spectroscopy measurements has been prepared using a process described in detail in the Supporting Information (SI, see Figures S1 and S2). Briefly, AuNS $(50 \pm 7 \mathrm{~nm})^{31}$ were caused to aggregate via nonspecific binding through stirring and then coated with 11-mercaptoundecanethiol (11MUA) to stabilize small aggregated structures. Dimers were extracted from the reaction mixture using electrophoretic separation. The UV-Vis spectrum of the re-suspended dimer sample shows a main peak at $530 \mathrm{~nm}$ due to the presence of monomers and a shoulder at $620 \mathrm{~nm}$, which matches well with a simulated dimer extinction spectrum (Figure 1A). Transmission electron microscopy (TEM) analysis (Figure 1B and Figures S3 and S4) confirms the specific enrichment of dimers, which constituted $23 \%$ of the sample with only $9 \%$ of larger aggregates present. Using high resolution TEM (Figure 1B, inset), the interparticle separation was found to be $1-2 \mathrm{~nm}$ for 
all measured dimers. Individual nanostructures were isolated for single-particle spectroscopy via

2 drop casting right after preparation although the dimer sample was stable in suspension for at

3 least a week at room temperature (Figure S5).
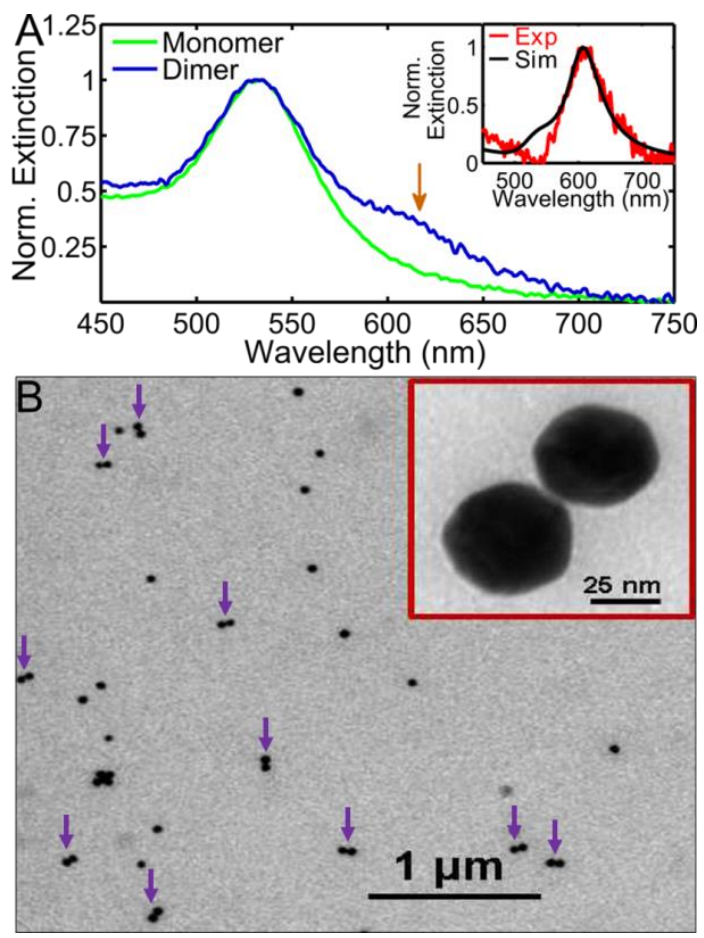

Figure 1. AuNS dimer characterization. (A) UV-Vis spectra of the purified and re-suspended monomer (green) and dimer (blue) sample. The orange arrow indicates the red-shifted extinction peak originating from plasmon coupling. Inset: Difference spectrum (red) obtained by subtracting the extinction spectrum of the monomer sample from the spectrum of the dimer sample is compared to a simulated dimer spectrum (black). (B) TEM image of the dimer sample with purple arrows indentifying individual AuNS dimers. Inset: High magnification TEM image of a representative AuNS dimer.

In the single-particle spectroscopy measurements, individual AuNS monomers and dimers were distinguished based on correlated scanning electron microscopy (SEM, FEI Quanta

7400 ESEM) using indexed quartz substrates or dark-field imaging with a color camera (Figure 2). A home-built microscope setup, as described in detail in Figure S6, allowed us to acquire

9 dark-field scattering (DFS) and PL images and spectra on the same sample area. A representative 
1

color image taken under transmitted light dark-field illumination with a digital single-lens reflex camera (DLSR) camera mounted directly to one of the microscope ports is shown in Figure 2A along with high-magnification SEM images of the corresponding nanostructures. Monomers and dimers can be clearly distinguished by the color of the scattered light, green and orange, respectively. Because the majority of the nanostructures were monomers and dimers (Figure 1B), color-based identification was easily achieved and greatly reduced the efforts in distinguishing and locating AuNS monomers and dimers, especially in the absence of correlated SEM data. In addition to color camera imaging, DFS images were acquired with an avalanche photodiode (APD) detector by using a pinhole in the microscope first image plane and scanning the sample. This modality enabled the positioning of individual nanostructures for spectroscopic investigations (see below). PL images were obtained in a similar configuration using a $488 \mathrm{~nm}$ continuous wave laser and appropriate dichroic and notch filters. With the help of the DSLR camera and SEM imaging (Figure 2A) of the same sample area, the identification of AuNS monomers and dimers was possible in the DFS (Figure 2B) and PL (Figure 2C) images, as indicated by the green and blue circles, respectively. Given the larger intensities of AuNS dimers, especially in DFS, and the enhanced sensitivity of the APD detector at red wavelengths, this correlated imaging was necessary even in regions that did not also contain one of the low percentage larger aggregates. 

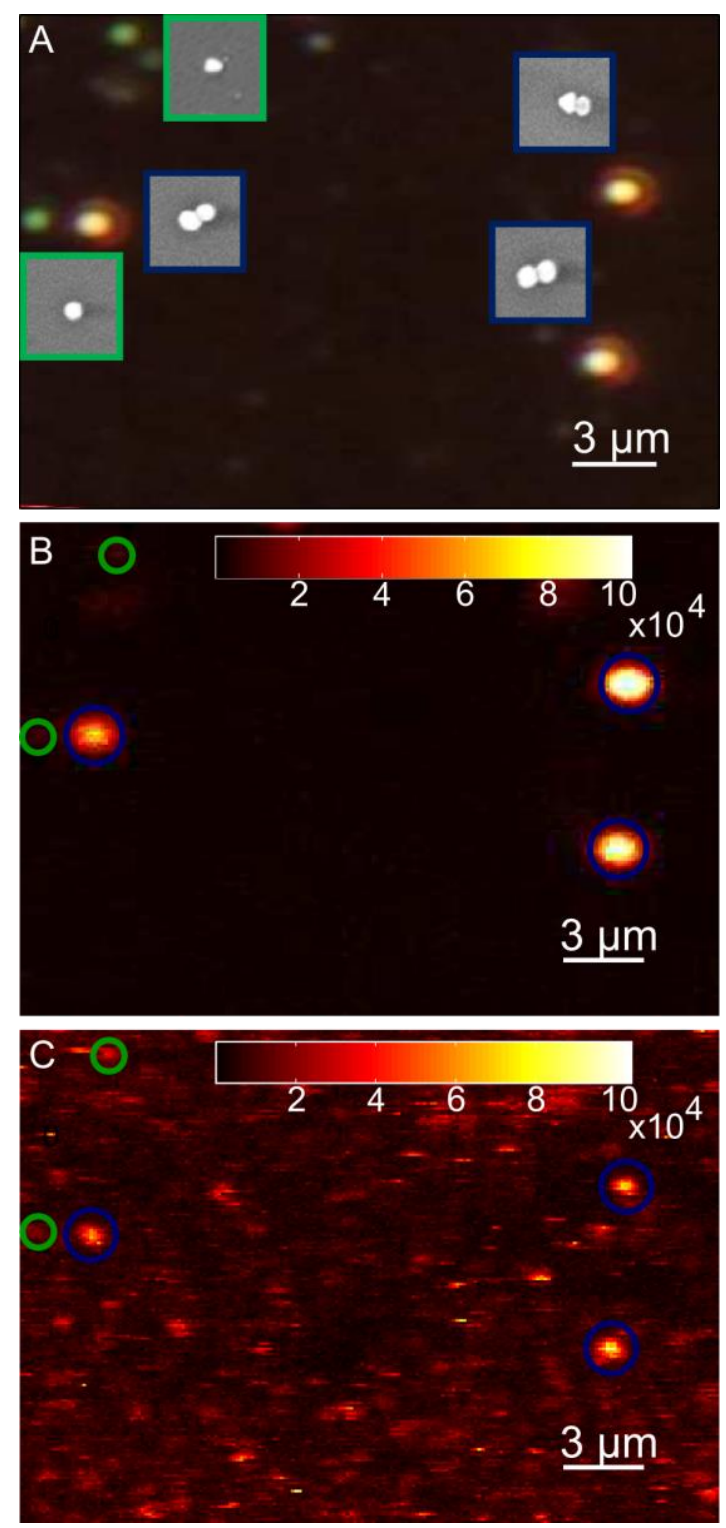

Figure 2. Correlated imaging of individual AuNS monomers and dimers. (A) DSLR camera image of the individual AuNS monomers and dimers on a quartz substrate observed under darkfield excitation. Correlated high-magnification SEM images are shown next to the particles. (B) and (C) show sample-scanned DFS and PL images of the same area as in (A), respectively. Monomers and dimers, as indicated by the green and blue circles, were located using the combined information from all images. Sample regions with larger aggregates were avoided as their increased DFS intensities made correlated imaging and identification of individual AuNS monomers and dimers almost impossible. PL images were recorded using a circularly polarized $488 \mathrm{~nm}$ laser with an intensity at the sample of $4.7 \mathrm{~kW} / \mathrm{cm}^{2}$ to avoid photothermal shape changes of mainly the dimers. Even though the signal-to-noise ratio in the PL images was reduced because of the low excitation intensity, it was still high enough to identify monomers and dimers for spectroscopic investigations (Figure 3). 
Single-particle spectra were collected by moving the sample to the location of a single AuNS monomer or dimer and switching the detection to a spectrometer equipped with a CCD camera. Figures $3 \mathrm{~A}$ and $3 \mathrm{~B}$ show the normalized DFS spectra of a representative AuNS monomer and dimer, respectively. The single DFS peak of the monomer, centered at $546 \mathrm{~nm}$, is the typical dipolar plasmon mode. In contrast, the DFS spectrum of the AuNS dimer shows two maxima as a result of the near-field capacitive coupling: a longitudinal polarized resonance at $650 \mathrm{~nm}$ and a much weaker transverse polarized resonance mode at $545 \mathrm{~nm}$. These results are in very good agreement with the well-studied scattering response of plasmonic dimers ${ }^{32,33}$ and can be understood in terms of two coupled dipoles, as illustrated by the surface charge plots simulated at the scattering maxima and shown as insets. The longitudinal mode arises from the in-phase coupling of the two dipoles along the dimer axis, creating an enhanced dipole and hence scattering cross section. This mode is usually referred to as being "super-radiant". The shorter wavelength transverse mode, where the dipoles are aligned vertically to the dimer axis, has a much weaker dipole as reflected in the smaller amplitude of the corresponding DFS peak and is often termed a "sub-radiant" mode. ${ }^{32}$

While the PL spectra show resonance maxima at similar spectral positions and the same polarization dependence, several differences exists between the DFS and PL spectra, as especially evident from the AuNS dimers. For comparison, the corresponding PL spectra of the same nanostructures are plotted along the DFS spectra in Figure 3. The PL spectra were corrected by the spectral sensitivity of the detection system (Figure S7). DFS spectra taken before and after the PL measurements ensured that the laser power was kept low enough at 4.7 $\mathrm{kW} / \mathrm{cm}^{2}$ to avoid photothermal damage, especially important for the closely spaced AuNSs comprising the dimers (Figure S8). The PL spectrum of the AuNS monomer peaks at $523 \mathrm{~nm}$, 
1

and its line shape resembles that of the DFS spectrum. The PL spectrum of the AuNS dimer also shows two resonance maxima at $527 \mathrm{~nm}$ and $634 \mathrm{~nm}$. Polarization dependent PL measurements (Figure S9) reveal that the lower and higher energy peaks are similarly polarized along and perpendicular to the long axis of dimer, respectively. The appearance of two coupled PL bands

for the AuNS dimer suggests a plasmonic origin of the PL and furthermore illustrates that PL

line shapes can be tailored through near-field coupling in specifically designed plasmonic molecules, just as is well-known for scattering. However, DFS and PL spectra are also different in two aspects: 1) for the low energy modes, the PL maxima are blueshifted by an average of $23 \pm 2$ and $22 \pm 2 \mathrm{~nm}$ for 25 AuNS monomers and 26 dimers, respectively. Although about 2 times larger in magnitude, this blueshift between PL and DFS spectra is consistent with previous PL studies of nanorods ${ }^{13,23} .2$ ) the relative intensities of the two dimer resonances do not match between PL and DFS. In the DFS spectra, the peak intensity of the super-radiant mode is approximately 6 times larger than that of the sub-radiant mode, while the lower energy mode in the PL spectra has a peak intensity that is on average just $90 \%$ of the higher energy mode emission. These differences will be discussed in more detail below.

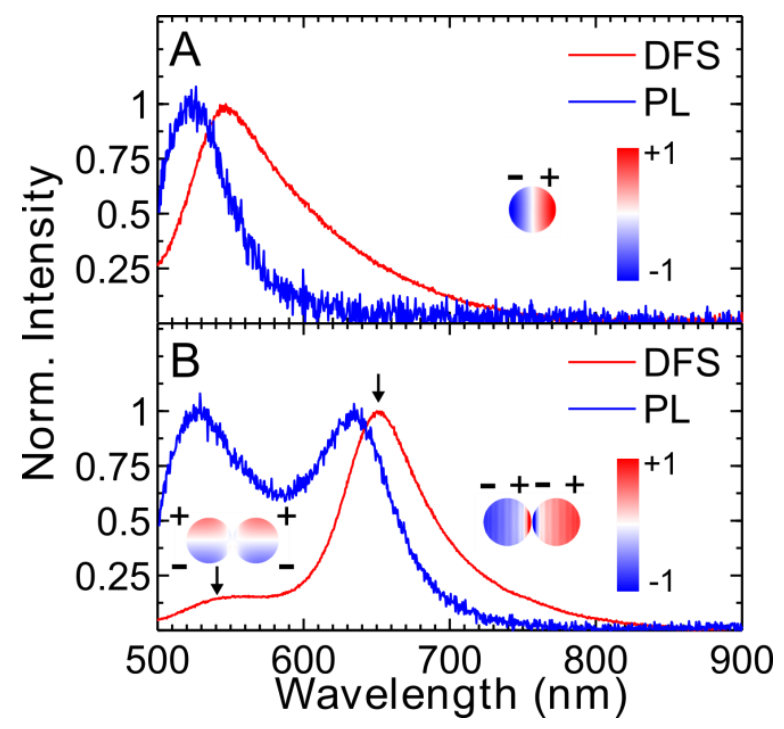


Figure 3. Representative single-particle DFS (red) and PL (blue) spectra of a AuNS monomer (A) and a AuNS dimer (B). DFS scattering spectra were measured using unpolarized transmitted light dark-field excitation, while the $488 \mathrm{~nm}$ excitation laser used to record the PL spectra was circularly polarized. The laser intensity at the sample was $4.7 \mathrm{~kW} / \mathrm{cm}^{2}$. A depolarizer in front of the spectrometer removed any polarization bias in the detection. Charge distributions simulated at the DFS resonance maxima are included as insets to identify the plasmon modes supported by these nanostructures.

1
Based on the single-particle imaging and spectroscopy demonstrated in Figures 2 and 3, we were able to calculate the PL quantum yields of AuNS monomers and dimers quantitatively, and found them to be about equal. The PL quantum yield from individual nanostructures was calculated taking the following factors into account: the $488 \mathrm{~nm}$ laser excitation intensity, the recorded emission intensity, the wavelength-dependent detection efficiency, and the calculated absorption cross sections at $488 \mathrm{~nm}$. Details for the PL quantum yield calculation can be found in the SI. $488 \mathrm{~nm}$ was chosen so that for both nanostructures the same interband transitions were excited and a direct comparison is valid, as evidence exists that the PL quantum yield depends on the type of transitions excited. ${ }^{14,15}$ The PL quantum yields of 25 AuNS monomers and 26 dimers are summarized in Figure 3. The average PL quantum yield of the monomers is $(1.92 \pm 0.72) \times 10^{-}$ ${ }^{6}$, in agreement with previous measurements of single AuNSs. ${ }^{34}$ The average value of the PL quantum yield measured for the AuNS dimers is basically the same with $(1.91 \pm 0.62) \times 10^{-6}$. Importantly, the distribution shows a similar spread and no rare cases with unusually high PL quantum yields were observed. 


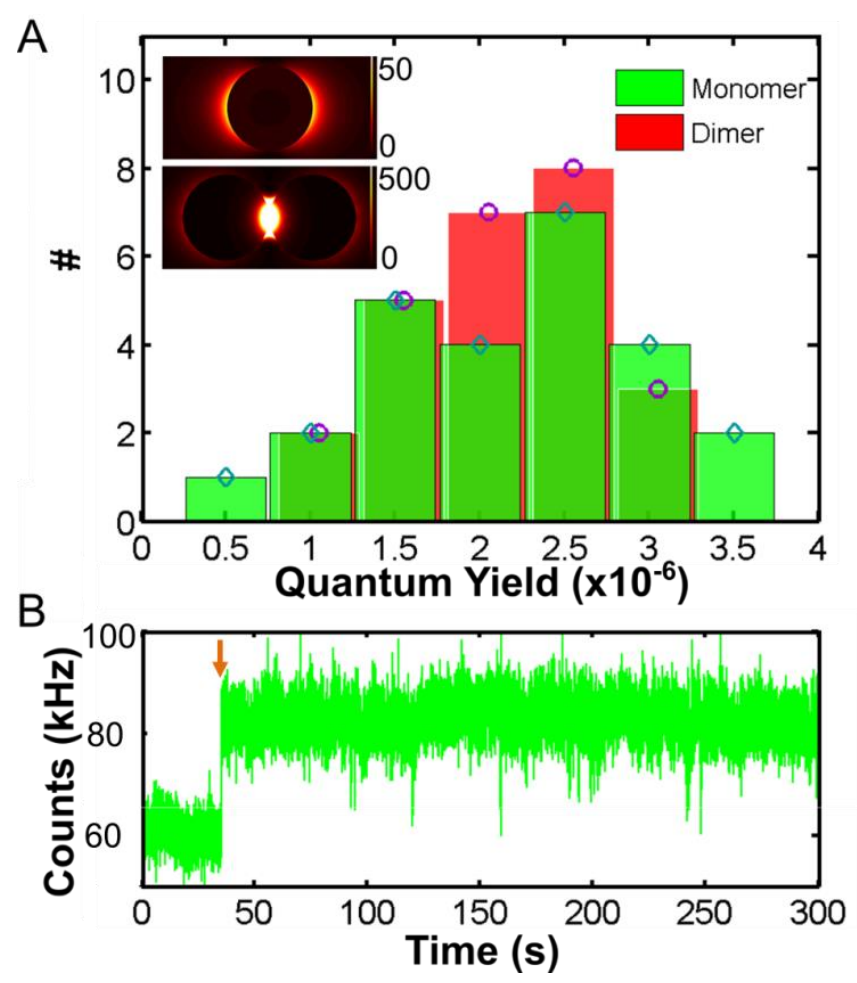

Figure 4. (A) Histograms of measured quantum yields for AuNS monomers (green) and dimers (red). Inset: Electromagnetic field enhancements $\left(|\mathrm{E}|^{2} /\left|\mathrm{E}_{0}\right|^{2}\right)$ for a monomer and a dimer calculated at $550 \mathrm{~nm}$ and $640 \mathrm{~nm}$, respectively. $\mathrm{E}_{0}$ is the incident electromagnetic field and $\mathrm{E}$ is the electric field around the nanostructure. Note the different intensity scales for monomer and dimer and that the electric field enhancement is even higher in the dimer gap than the maximum value of the scale in order to outline the positions of the AuNSs. (B) Photon count rate versus time for PL from a AuNS monomer. The orange arrow indicates the time when the single AuNS monomer was moved over the excitation beam (intensity of $4.7 \mathrm{~kW} / \mathrm{cm}^{2}$ ). Neither photoblinking nor photobleaching were observed over the time course of 5 min shown here.

The absence of an enhanced PL from the AuNS dimer allows us to exclude as possible

3 mechanisms antenna-enhanced emission from surface ligands ${ }^{19,20}$ and intrinsic small clusters ${ }^{21}$, considering that both monomers and dimer originate from the same initial AuNS solution and were encapsulated in similar 11-MUA shells during the dimer enrichment procedure. This

6 conclusion is based on the fact that the AuNS dimer is in general a much stronger antenna. The

7 local electric field enhancement of a dimer with a $1 \mathrm{~nm}$ interparticle gap was calculated to be 2-3

8 orders of magnitude larger than that of a monomer when averaged over the cross sectional area 
1

as illustrated in the insets of Figure 4A. If molecular emitters were distributed evenly over the dimer antenna, a significantly larger average PL quantum yield would be expected even when considering a possible change in spectral overlap with relatively sharp molecular resonances. If, on the other hand, only a few emitters were present at random position, a significantly broader distribution of PL quantum yields, apart from a possible change of its average value, should have been measured. Furthermore, photobleaching especially for our samples in air should be observed if only a few molecular impurities were responsible for the measured PL. The PL emission intensity of a single AuNS monomer was, however, constant for over 4 min as shown in Figure 4B. Considering both the PL quantum yield distributions and the dimer PL spectrum itself, our data also suggest that plasmon enhanced intrinsic electron-hole pair recombinations in Au are likely not the main origin of the measured PL. ${ }^{6-11}$ The dimer PL spectrum shows unique coupled PL modes and is clearly distinct from scattering, which is about three times higher for the dimer than the monomer when analyzing the integrated areas. However, increased nonradiative damping due to near-field coupling could reduce the PL quantum of the dimers, as suggested for Au nanoparticle arrays fabricated by electron-beam lithography. ${ }^{18}$

As we found no strong evidence here for a local electric field dominated PL mechanism, we turn our attention to the suggested radiative decay of surface plasmons or plasmon emission. $^{12-18}$ Plasmon emission involves the excitation of a surface plasmon by an excited electron-hole pair, where the electron-hole pair is initially created either through direct excitation (i.e. interband transitions) or from the decay of an excited plasmon (Figure 5A). This mechanism was initially proposed for $\mathrm{Au}$ nanorods based on excitation wavelength and excitation polarization independent emission with a spectral position, line shape, and polarization matching those of the DFS spectrum ${ }^{14}$ and has been supported by more recent studies. ${ }^{3,13,15-17}$ Direct 
1

surface plasmon emission has also been proposed feasible and theoretically verified for the visible light emission from a scanning tunneling microscope probing silver films. ${ }^{35,36}$ While a small blueshift between PL and DFS spectra was previously noted for Au nanorods, ${ }^{13,23}$ it was mainly ignored as it could not be easily explained. However, the differences observed here in the PL and DFS spectra for the AuNS monomers and dimers (Figure 3) are much more significant as already mentioned above. As the step of surface plasmon excitation by an excited electron-hole pair within the suggested mechanism represents the reverse process of surface plasmon decay ${ }^{37}$, the PL spectrum should also be compared to the absorption spectrum, which quantifies to a first approximation the non-radiative relaxation channel of hot carrier generation ${ }^{38-40}$. Pure absorption spectra of single nanostructures with sizes larger than a few tens of nanometers are difficult to obtain though, and we therefore resort to simulated absorption spectra using Mie theory for monomers and Generalized Mie theory (GMT) for dimers (see SI for simulation details).

Although we find in general a better spectral match between PL and absorption spectra, we must conclude that the PL line shape is unique and likely arises from a combination of factors related to non-radiative (proportional to absorption) and radiative (proportional to scattering) properties. Figures 5B and 5C compare simulated absorption and scattering spectra of a AuNS monomer $(5 \mathrm{~B})$ and a dimer $(5 \mathrm{C})$. The scattering resonance maxima are $547 \mathrm{~nm}$ for the monomer and $532 \mathrm{~nm}$ and $652 \mathrm{~nm}$ for the dimer with a calculated peak intensity ratio for the super- and sub-radiant modes of $\sim 4$. The simulated scattering spectra hence match well with the experimental DFS spectra and allow for a comparison between simulated absorption spectra and measured PL. Considering the predicted blueshifts from scattering and the increased relative absorption intensity of the sub-radiant plasmon mode for the dimer in the simulations, the PL spectra resemble more closely the corresponding AuNS monomer and dimer absorption spectra. 
However, the blueshift is only $6 \mathrm{~nm}$ for the low energy modes of both monomers and dimers, much less than the $>20 \mathrm{~nm}$ peak shifts observed experimentally for the PL. In addition, Figure

$35 \mathrm{D}$ plots the peak area ratios for the two spectral bands of the AuNS dimers, as obtained by

4 fitting each spectrum with the sum of two Lorentzian curves and integrating the areas of each

5 peak, against the resonance maximum of the corresponding lower energy peak. For the PL, we 6 observe experimentally a decrease of this ratio with increased plasmon coupling (longer

7 resonance wavelength). The same ratio was calculated from simulated absorption spectra of 8 dimers where we randomly varied the AuNS diameter over a range of $50 \pm 5 \mathrm{~nm}$ and the gap size 9 over a range of $1.5 \pm 0.5 \mathrm{~nm}$, consistent with TEM characterization of the sample (see SI). While 10 the ratios for absorption are similar in magnitude, no dependence on the resonance wavelength of 11 the super-radiant mode is seen. This trend for absorption is reasonable because the absorption 12 peak area is proportional to the total number of electrons. ${ }^{39,41}$ Varying the interparticle distance 13 does not change the total amount of electrons, and varying the AuNS size adds electrons to both 14 higher and lower energy modes. We must therefore conclude that, in addition to non-radiative 15 processes such as hot electron generation, other factors influence the PL spectrum, such as 16 coupling to bright photonic states that facilitate far-field emission of a plasmon. The multi-step

17 mechanism in Figure 5A illustrates this suggested interplay between non-radiative pathways 18 (excitation of plasmons by hot carriers and decay of plasmons into them as indicated by the black 19 arrows) and radiative properties (light emission as indicated by the wavy green and red arrows). 

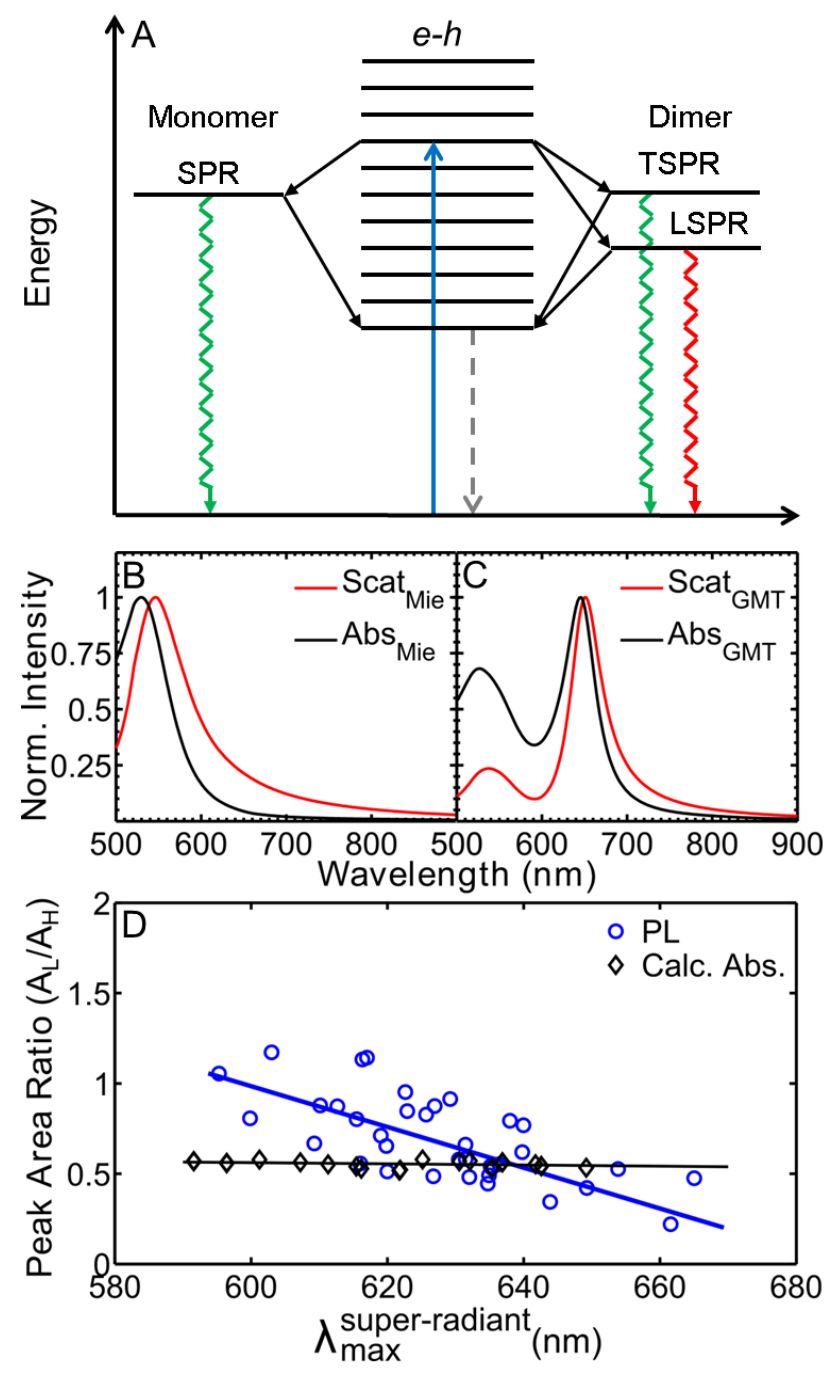

Figure 5. (A) Multi-step PL mechanism: $488 \mathrm{~nm}$ excitation (blue arrow) creates electron-hole pairs (e-h) via interband excitation. The majority of these excited carries relaxes non-radiatively (gray dotted arrow), but some can create surface plasmons (black arrows). The monomer supports only one surface plasmon resonance (SPR), while the dimer has a transverse polarized mode (TSPR) and a lower energy longitudinal polarized mode (LSPR). These surface plasmons either convert back into electron-hole pairs (black arrows) or decay radiatively (green and red wavy arrows) giving rise to PL. Simulated normalized scattering (red) and absorption (black) spectra of a AuNS monomer (B) and dimer (C). For the AuNS monomer, Mie theory was used: AuNS diameter $=50 \mathrm{~nm}$. For the AuNS dimer, GMT was used: AuNS diameter $=53 \mathrm{~nm}$ for both constituents with an interparticle gap of $1 \mathrm{~nm}$. (D) Peak area ratios for the two spectral bands of the AuNS dimers as a function of the corresponding resonance wavelength of the lower energy mode (blue circles: PL; black diamonds: calculated absorption). The lines are linear fits shown as visual guides only. $A_{L}$ is the integrated area of the lower energy peak and $A_{H}$ is the integrated area of the higher energy peak. A homogeneous dielectric constant of 1.25 was assumed for the surrounding medium in all calculations. 
CONCLUSIONS

In summary, we have prepared an enriched AuNS dimer sample that allowed us to

4

6
investigate the PL of individual strongly coupled plasmonic molecules. We found that the PL spectrum is tuned by plasmon coupling and that the quantum yields of dimers and constituent 50 $\mathrm{nm}$ AuNS monomers are the same, indicating that the enhanced electric fields around the dimers do not strongly influence the PL in contrast to several proposed PL mechanisms. We have furthermore compared the measured AuNS monomer and dimer PL spectra with the corresponding scattering and absorption spectra, which are each proportional to important steps that are thought to play a role in the proposed plasmon emission mechanism, i.e. far-field radiation from bright states and generation of hot electrons. Our results are consistent with direct plasmon emission, in which the plasmon is driven by generated hot electrons. A more quantitative theory considering the density of electronic and plasmonic states is desperately needed to gain further understanding of the PL from plasmonic nanostructures as well as a recently reported anti-Stokes emission. ${ }^{42,43}$

\section{METHODS}

Materials. Citrate capped AuNSs with nominal diameters of $50 \mathrm{~nm}$ were purchased from BBI solutions (Cardiff, UK). Independent transmission electron microscopy (TEM) characterization yielded a size dispersion of $51 \pm 7 \mathrm{~nm}^{31}$. 11-mercaptoundecanoic (11-MUA) with 95\% purity was obtained from Sigma-Aldrich (St. Louis, CA). Acetonitrile was purchased from VWR International (Radnor, PA). Deionized water was obtained from an ultrapure water system from EMD Millipore (Billerica, MA).

Synthesis of AuNS Dimer. The synthetic procedure for forming AuNS dimers is illustrated in Figure S1. $500 \mu \mathrm{L}$ of AuNS solution were washed with deionized water once and then concentrated to a volume of $50 \mu \mathrm{L}$ by centrifugation at $1400 \mathrm{rcf}$ for $5 \mathrm{~min}$. $200 \mu \mathrm{L}$ acetonitrile were added to the AuNS suspension, and this solution was stirred for $30 \mathrm{~min}$ followed by addition of $5 \mu \mathrm{L}$ of $10 \mathrm{mM} \mathrm{11-MUA.} \mathrm{After} \mathrm{another} 30 \mathrm{~min}$ of stirring, the solution 
was centrifuged to remove the supernatant and finally re-suspended in $500 \mu \mathrm{L}$ of deionized water. Aggregates consisting of different numbers of AuNSs were separated and purified by gel electrophoresis using a horizontal gel electrophoresis system from Bio-Rad (Hercules, CA). Specifically, the AuNS mixtures were first concentrated into a total volume of $50 \mu \mathrm{L}$, followed by addition of $10 \mu \mathrm{L}$ aqueous sucrose solution ( $40 \mathrm{w} / \mathrm{v} \%$ ). The samples were mixed well and then loaded onto $1 \%$ agarose gel. The gel was run at a constant potential of $135 \mathrm{~V}$ for $30 \mathrm{~min}$ with a running buffer of $0.5 \mathrm{X}$ Tris-Acetate-EDTA (TAE). The different bands in the gel were manually cut off with a razor blade before the AuNS monomers, dimers, and aggregates were extracted via a published eletro-elution method $^{44}$ under twice the electrophoresis voltage. The final step involved concentration of the extracted products by centrifugation. TEM images were taken using a JEOL 1230 to characterize AuNS aggregates after reaction and purification (Figure S3).

Sample Preparation for Single Particle Measurement. Samples with an appropriate density for single-particle spectroscopy were obtained by drop-casting the AuNS dimer solution onto the patterned substrates. Cleaned $25.4 \mathrm{~mm}$ quartz slides (AdValue Tech, Tucson, AZ) with $1 \mathrm{~mm}$ thickness were used as substrates instead of glass because of their lower background. A gold identification pattern was created on the substrates using an electron-beam evaporator and a copper TEM grid as a mask. ${ }^{45}$ SEM was performed after single-particle spectroscopy measurement using an FEI Quanta 400 ESEM.

Characterization with Single Particle Spectroscopy. The single-particle experiments were performed on a home-built instrument (Figure S6) based on an inverted Zeiss microscope described previously ${ }^{13}$ with slight modifications. Scattering spectra were obtained using a halogen lamp for unpolarized excitation and an oil immersion dark-field condenser. Scattered light was collected by a $50 \mathrm{X}$ air-spaced objective with a numerical aperture of 0.8 and then passed through a pinhole at the first image plane, effectively achieving confocal conditions. A scanning stage (P-517.3CL, Physik Instrumente, Karlsruhe/Palmbach, Germany) was used to scan the sample and to construct a DFS image. An avalanche photodiode (APD, SPCM-AQRH15, Perkin-Elmer, Waltham, MA) was employed to record the scattered light intensity. Singleparticle spectra were obtained by positioning the sample so that an isolated nanosctructure was reimaged onto the pinhole and the signal was re-directed to a spectrometer (SP2150, Princeton Instruments, Trenton, NJ) equipped with a CCD camera (PIXIS 400BR, Princeton Instruments, Trenton, NJ). Scattering spectra were corrected with a white light standard. PL spectroscopy was conducted in a similar way except that a collimated $488 \mathrm{~nm}$ laser beam from an $\mathrm{Ar}^{+}$laser (Stellar-Pro, Modu-Laser, Centerville, UT) was used as the excitation source. The laser power was kept below $4.7 \mathrm{~kW} / \mathrm{cm}^{2}$ measured at the sample. We estimated the size of the focused laser beam to have a full width at half maximum of $420 \mathrm{~nm}$. A dichroic filter (Chroma, Bellows Falls, VT), notch filter (Semrock, Rochester, NY), and a $496 \mathrm{~nm}$ long-pass filter (Semrock, Rochester, NY) were used to separate excitation from emission. A quarter waveplate was used to generate circularly polarized laser light. A depolarizer in front of the spectrometer was used to remove any polarization bias in the detection path. The PL spectrum was corrected for background emission from the substrate and by the wavelength-dependent sensitivity of the microscope setup (Figure S7). 
3 ASSOCIATED CONTENT

4 Supporting Information

5 Details regarding experimental \& computational methods and data analysis. This material is

6 available free of charge via the Internet at http://pubs.acs.org.

7

8 AUTHOR INFORMATION

9 Corresponding Author

$10 *$ slink@rice.edu

$11 *$ cflandes@rice.edu

12 Notes

13 The authors declare no competing financial interests.

14

15 ACKNOWLEDGEMENTS

16 This work was funded by the Robert A. Welch Foundation (Grants C-1664 and C-1787), the

17 National Science Foundation (Grant CHE-0955286), and the Army (MURI W911NF-12-1-

18 0407). C.P.B. and A.H. acknowledge support from the National Science Foundation through a

19 Graduate Research Fellowship (0940902). We thank M. Yorulmaz, A. Manjavacas and P.

20 Nordlander for useful discussions.

21

22 
References:

(1) Juette, M. F.; Terry, D. S.; Wasserman, M. R.; Zhou, Z.; Altman, R. B.; Zheng, Q.; Blanchard, S. C. Curr. Opin. Chem. Biol. 2014, 20, 103-111.

(2) Xia, T.; Li, N.; Fang, X. H. Annu. Rev. Phys. Chem. 2013, 64, 459-480.

(3) Zhang, T. Y.; Shen, H. M.; Lu, G. W.; Liu, J.; He, Y. B.; Wang, Y. W.; Gong, Q. H. Adv. Opt. Mater. 2013, 1, 335-342.

(4) Xu, Z. C.; Huang, X. Y.; Dong, C. Q.; Ren, J. C. Microchim. Acta 2014, 181, 723-730.

(5) Stender, A. S.; Marchuk, K.; Liu, C.; Sander, S.; Meyer, M. W.; Smith, E. A.; Neupane, B.; Wang, G. F.; Li, J. J.; Cheng, J. X.; Huang, B.; Fang, N. Chem. Rev. 2013, 113, 2469-2527.

(6) Mooradia, A. Phys. Rev. Lett. 1969, 22, 185-187.

(7) Mohamed, M. B.; Volkov, V.; Link, S.; El-Sayed, M. A. Chem. Phys. Lett. 2000, 317, 517523.

(8) Lumdee, C.; Yun, B.; Kik, P. G. ACS Photonics 2014, 1, 1224-1230.

(9) Apell, P.; Monreal, R.; Lundqvist, S. Phys. Scripta 1988, 38, 174-179.

(10) Boyd, G. T.; Yu, Z. H.; Shen, Y. R. Phys. Rev. B 1986, 33, 7923-7936.

(11) Beversluis, M. R.; Bouhelier, A.; Novotny, L. Phys. Rev. B 2003, 68, 115433.

(12) Dulkeith, E.; Niedereichholz, T.; Klar, T. A.; Feldmann, J.; Von Plessen, G.; Gittins, D. I.; Mayya, K. S.; Caruso, F. Phys. Rev. B 2004, 70, 205424.

(13) Fang, Y.; Chang, W. S.; Willingham, B.; Swanglap, P.; Dominguez-Medina, S.; Link, S. ACS Nano 2012, 6, 7177-7184.

(14) Tcherniak, A.; Dominguez-Medina, S.; Chang, W. S.; Swanglap, P.; Slaughter, L. S.; Landes, C. F.; Link, S. J. Phys. Chem. C 2011, 115, 15938-15949.

(15) Zhang, T. Y.; Lu, G. W.; Shen, H. M.; Shi, K. B.; Jiang, Y. Y.; Xu, D. S.; Gong, Q. H. Scientific Reports 2014, 4, 3867.

(16) Hu, H. L.; Duan, H. G.; Yang, J. K. W.; Shen, Z. X. ACS Nano 2012, 6, 10147-10155.

(17) Wackenhut, F.; Failla, A. V.; Meixner, A. J. J. Phys. Chem. C 2013, 117, 17870-17877.

(18) Walsh, G. F.; Dal Negro, L. Nano Lett. 2013, 13, 786-792.

(19) Goldys, E. M.; Sobhan, M. A. Adv. Funct. Mater. 2012, 22, 1906-1913.

(20) Chang, H. Y.; Chang, H. T.; Hung, Y. L.; Hsiung, T. M.; Lin, Y. W.; Huang, C. C. RSC Adv. 2013, 3, 4588-4597.

(21) Zhou, C.; Yu, J.; Qin, Y. P.; Zheng, J. Nanoscale 2012, 4, 4228-4233.

(22) Huang, J. Y.; Wang, W.; Murphy, C. J.; Cahill, D. G. Proc. Natl. Acad. Sci. 2014, 111, 906911.

(23) Yorulmaz, M.; Khatua, S.; Zijlstra, P.; Gaiduk, A.; Orrit, M. Nano Lett. 2012, 12, 43854391.

(24) Rao, W.; Li, Q.; Wang, Y.; Li, T.; Wu, L. ACS Nano 2015, DOI: 10.1021/nn506689b.

(25) Klimov, V. V.; Guzatov, D. V. Appl. Phys. a-Mater. 2007, 89, 305-314.

(26) Wang, H.; Brandl, D. W.; Nordlander, P.; Halas, N. J. Acc. Chem. Res. 2007, 40, 53-62.

(27) Fromm, D. P.; Sundaramurthy, A.; Schuck, P. J.; Kino, G.; Moerner, W. E. Nano Lett. 2004, 4, 957-961.

(28) Muhlschlegel, P.; Eisler, H. J.; Martin, O. J. F.; Hecht, B.; Pohl, D. W. Science 2005, 308, 1607-1609.

(29) Muskens, O. L.; Giannini, V.; Sanchez-Gil, J. A.; Rivas, J. G. Nano Lett. 2007, 7, 2871 2875. 
(30) Indrasekara, A. S. D. S.; Paladini, B. J.; Naczynski, D. J.; Starovoytov, V.; Moghe, P. V.; Fabris, L. Adv. Healthc. Mater. 2013, 2, 1370-1376.

(31) Dominguez-Medina, S.; Blankenburg, J.; Olson, J.; Landes, C. F.; Link, S. ACS Sustain. Chem. Eng. 2013, 1, 833-842.

(32) Willingham, B.; Brandl, D. W.; Nordlander, P. Appl. Phys. B: Lasers Opt. 2008, 93, 209216.

(33) Thacker, V. V.; Herrmann, L. O.; Sigle, D. O.; Zhang, T.; Liedl, T.; Baumberg, J. J.; Keyser, U. F. Nat. Commun. 2014, 5, 3448

(34) Gaiduk, A.; Yorulmaz, M.; Orrit, M. Chemphyschem 2011, 12, 1536-1541.

(35) Uehara, Y.; Kimura, Y.; Ushioda, S.; Takeuchi, K. Jpn. J. Appl. Phys. 1 1992, 31, 24652469.

(36) Iida, W.; Ahamed, J. U.; Katano, S.; Uehara, Y. Jpn. J. Appl. Phys. 2011, 50, 095201.

(37) Sonnichsen, C.; Franzl, T.; Wilk, T.; von Plessen, G.; Feldmann, J.; Wilson, O.; Mulvaney, P. Phys. Rev. Lett. 2002, 88, 077402

(38) Manjavacas, A.; Liu, J. G.; Kulkarni, V.; Nordlander, P. ACS Nano 2014, 8, 7630-7638.

(39) Clavero, C. Nat. Photonics 2014, 8, 95-103.

(40) Govorov, A. O.; Zhang, H.; Demir, H. V.; Gun'ko, Y. K. Nano Today 2014, 9, 85-101.

(41) Malloci, G.; Cappellini, G.; Mulas, G.; Mattoni, A. Chem. Phys. 2011, 384, 19-27.

(42) He, Y.; Xia, K.; Lu, G.; Shen, H.; Cheng, Y.; Liu, Y.; Shi, K.; Xiao, Y.; Gong, Q.

Nanoscale 2015, 7, 577-582.

(43) Neupane, B.; Zhao, L. Y.; Wang, G. F. Nano Lett. 2013, 13, 4087-4092.

(44) Reinhard, B. M.; Sheikholeslami, S.; Mastroianni, A.; Alivisatos, A. P.; Liphardt, J. Proc.

Natl. Acad. Sci. 2007, 104, 2667-2672.

(45) Nehl, C. L.; Grady, N. K.; Goodrich, G. P.; Tam, F.; Halas, N. J.; Hafner, J. H. Nano Lett. 2004, 4, 2355-2359. 